\title{
QoS Routing Method Considering Congestion in WMSNs
}

\author{
Si-Yeong Bae ${ }^{1}$, Sung-Keun Lee ${ }^{2}{ }_{\dagger}$, Jin-Gwang Koh $^{1}$ and Kyoung-Wook Park ${ }^{3}$ \\ ${ }^{1}$ Department of Computer Science, Sunchon National University, Korea \\ ${ }^{2}$ Department of Multimedia Engineering, Sunchon National University, Korea \\ ${ }^{3}$ Division of Culture Contents, Chonnam National University, Korea
}

\{bsy233,kjg\}@sunchon.ac.kr,sklee@sunchon.ac.kr,zergadiss73@chonnam.ac.kr

\begin{abstract}
In Wireless multimedia sensor networks (WMSNs), nêm metrics are required in order to accurately measure and evaluate link quality of nodes, residing along a routing path comprehensively considering end-to-end delay, throughtu and radio interference. Moreover, it is important to develop a routing algorithm that can find an optimal path to meet a QoS requirement based on the metrics. This study presents a QoS routing method taking into account congestion, which is aimed at mimizing endo-end delay and increasing throughput. With a use of congestion Control technique and least cost-based routing mechanism, the proposed routing method increases packet transmission rate whereas it reduces packet loss and delay. Ultimetely, it contributes to longer network lifetime of sensor networks, which could result to bffering better reliability of the network.
\end{abstract}

Keywords: QoS, WMSN, Link Quality, Congestion Control, Routing Protocol

\section{Introduction}

Most existing routing protocols for wireless sensor networks (WSNs) are considered to be data-centric protocol. Those protocols are designed to guarantee high energy efficiency and enhance network scalability Since WMSNs are required to deliver a huge amount of multimedia data in timely manner due to their nature, both of low data latency and high throughput are crucial requirements in WMSNs. For achieving these goals, new metrics are needed to accurately estimate quality of each link residing along the routing path comprehensively considering several factors such as delay, throughput and radio interference. Based on the new metrics, devising an efficient routing algorithm is also considered to be important 11 order to meet a given QoS requirement [1-3].

This paper proposes QoS routing scheme taking into account network congestion to enhance throughput as well as to minimize end-to-end delay in WMSNs. The proposed method determines an optimal path with lowest cost using traffic priority, link quality, residual energy of neighbor nodes and distance between nodes. Ultimately, the suggested protocol contributes to avoid the energy depletion problems of the nodes which may be preferred by the routing protocol due to their position. In addition, the proposed method enables to utilize an alternate path in an occurrence of faulty links by applying hop-by-hop congestion control based on priority. The alternate path can realize a reliable data transmission and minimize unnecessary retransmission due to data loss.

\footnotetext{
$\dagger$ Corresponding Author
} 


\section{QoS Routing Method considering Congestion}

\subsection{Routing Protocol Algorithm for Support QoS}

Source node establishes a path depending on priority field value marked in packet. Traditional protocol tends to select the shortest path when transmitting data. In other words, some nodes along the shortest path are frequently used to send data. Therefore, it shows poor energy efficiency since the nodes excluding the shortest path mostly stay in idle state. Moreover, network lifetime becomes short, which could lead to node failure due to power shortage. Consequently, network path is blocked and reliability is also damaged. The proposed routing protocol algorithm distributes network traffic into different paths by classifying packets into green, red and yellow packet. Green packet takes the shortest path to aim for high quality transmission, while red packet takes an alternate path eonsidering residual energy. Yellow packet, at the middle of green ând red, determines the path considering middle level of distance and quality.

Routing table suggested by this paper consists of following fields. The priority consists of 3 steps of Green, Yellow and Red. A next node dengtes a node which would receive the sensing data, while an alternate next node means a node which would be alternatively used in case that congestion is identified in main node Hop countemeans the number of hop from sink. The residual energy means a remaining energy of nodew which is to receive the sensing data. The neighbor node means the position of node which is to receive the sensing data. The path cost means the path costs required to sink node by packet priority.

Assume that the networks are dense) and there are several paths between two nodes. The path cost required for node $a$ to select the next node $b$ in order to transmit the packet whose priority is pri to sink node could be obtained by following equation 1 .

$$
C_{a}^{p r i}=\min _{b \in N_{a}}\left\{\alpha_{\text {pri }}\left(\frac{d_{B_{x}}+1}{d_{a, y}+1}-0.5\right) \times \beta_{p r i}\left(1-\frac{L Q I_{b}}{256}\right) \times \gamma_{p r i}\left(1-\frac{e_{b}^{r e s}}{e_{b}^{\text {init }}}\right)+C_{b}^{p r i}\right\}
$$

where pri denoles priority mark, pri $\in\{$ Green, Yello, Red $\}, N_{a}$ is a set of neighbor nodes of node $a . d_{a y}$ denotes hop distance from node $a$ to sink node $y . d_{b y}$ denotes hop distance between node $b$ and sink node $y . e_{b}^{\text {init }}$ is an initial energy level of node $b$, and $e_{b}^{r e s}$ is residual energy of node $b . L Q I_{b}$ is link quality value for node $b$, ranging from 0 to 255 . $\left(\frac{d_{b y}+1}{d_{a y}+1}-0.5\right),\left(1-\frac{L Q I_{b}}{256}\right)$ and $\left(1-\frac{e_{b}^{\text {res }}}{e_{b}^{\text {init }}}\right)$ denote hop distance, link quality and cost depending energy remainder respectively, ranging from 0 to 1.

$\alpha_{p r i}, \beta_{p r i}$ and $\gamma_{p r i}$ are weighted value differently set depending on packet's priority. Since green packet has highest priority, hop counts and link quality should be considered rather than remaining energy when setting the weighted values. If the nodes have the same hop counts, link quality needs to be considered subsequently. Therefore, $\alpha_{\text {Green }}$ and $\beta_{\text {Green }}$ is supposed to set to low value, whereas $\gamma_{\text {Green }}$ is set to high value. Yellow packet has middle level of priority. Therefore, hop count, link quality and remaining energy should be considered in balanced manner. Finally, red packet has the lowest priority, hence residual energy needs to be considered first rather than hop count and link quality. In order to minimize energy consumption, $\gamma_{\text {Red }}$ is set to low weighted value, whereas $\alpha_{\text {Red }}$ and $\beta_{\text {Red }}$ are set to be high. 


\subsection{Congestion Control}

In sensor networks, congestion might happen because the nodes consisting of network tend to generate significant amount of data. Once congestion occurs in network, data loss is inevitable due to packet collision or queue overflow resulting from competition in gaining limited wireless radio resources. Focusing on these causes, this paper suggests congestion control mechanism so as to guarantee reliable data transmission as well as to minimize unnecessary retransmission in wireless environment.

Congestion detection uses congestion degree being defined by using average packet service time and average packet inter-arrival time in Priority-based Congestion Cohtrol in Wireless Sensor Network (PCCP) [4] in order to determine congestion situration.

Packet inter-arrival time means time interval between two packets being sequentially arrived in one node $\left(t_{a}^{i}\right)$ and packet service time means duration from the time of arriving at MAC layer to the time when last bit of packet is transmitted $\left(t_{s}^{i}\right)$. Based on $t_{a}^{i}$ and $t_{s}^{i}$, congestion degree $\left(C D_{i}\right)$ is as following equation (2).

$$
C D_{i}=t_{a r}^{i} t_{s}^{i}
$$

In this paper, congestion situation is identified considering congestion degree and buffer occupancy at the same time androyting path (ff)node is established.

Figure 1 shows congestion control algorithm, where $C D_{i}$ denotes a degree of congestion of node $i$ and $q_{i}$ denotes abuffer occupancy rate of node $i$.

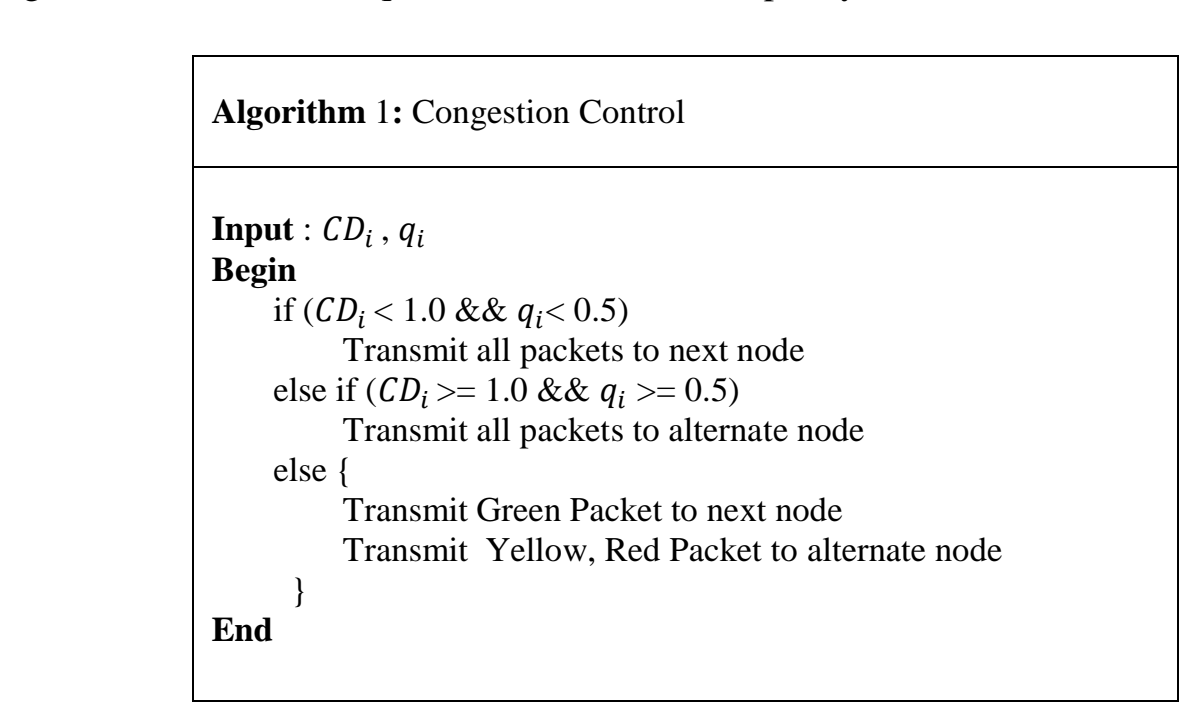

Figure 1. Congestion Control Algorithm

In case that $\mathrm{CD}_{\mathrm{i}}<1.0$ and $\mathrm{q}_{\mathrm{i}}<0.5$, as congestion situation was not taken place and buffer occupancy was not high, such case is considered that its data flow is smooth. At this time, transmission is made to determined next node.

In case that $C D_{i}>=1.0$ and $q_{i}>=0.5$, current situation is excessively congested condition and in this case, packet will be discarded as buffer overflow is taken place. Therefore, all the packets are transmitted to alternate node. 
As $\mathrm{CD}_{\mathrm{i}}$ value is smaller than $1, \mathrm{q}_{\mathrm{i}}$ is over $50 \%$ and buffer occupancy of node $\mathrm{i}$ is below $50 \%$, it is expected that buffer occupancy based on currently low congestion degree would be gradually decreased. However, occurrence possibility of packet drop by buffer overflow of node is increased by just slight increase of traffic due to high buffer occupancy. In case that $C D_{i}$ is bigger than 1 and $q_{i}$ is below $50 \%$, buffer occupancy is low but due to high congestion degree, buffer occupancy will be gradually increased and occurrence of congestion is highly likely to be taken place shortly. Therefore, based on traffic priority, Green packet shall be transmitted to main node and Yellow, Red packet shall be transmitted to alternate node.

\subsection{QoS Routing Method considering Congestion}

The QoS routing method with considering congestion in network can support differentiated service by establishing an optimal path with least cost using LQI value which is useful for identifying hop count toward sink node, residual energy of neighbor nodes and link quality. Additionally, the proposed method is capable of taking an alternate path in an occurrence of faulty link along the path through hop-by-hop congestion control in conjunction with priority of nodes. The alternative path can minimize unnecessary data retransmission that might happen due to congestion in primary path. As a result, reliable data transmission can be achieved.

The QoS routing algorithm with considering congestion is shown in Figure 2.

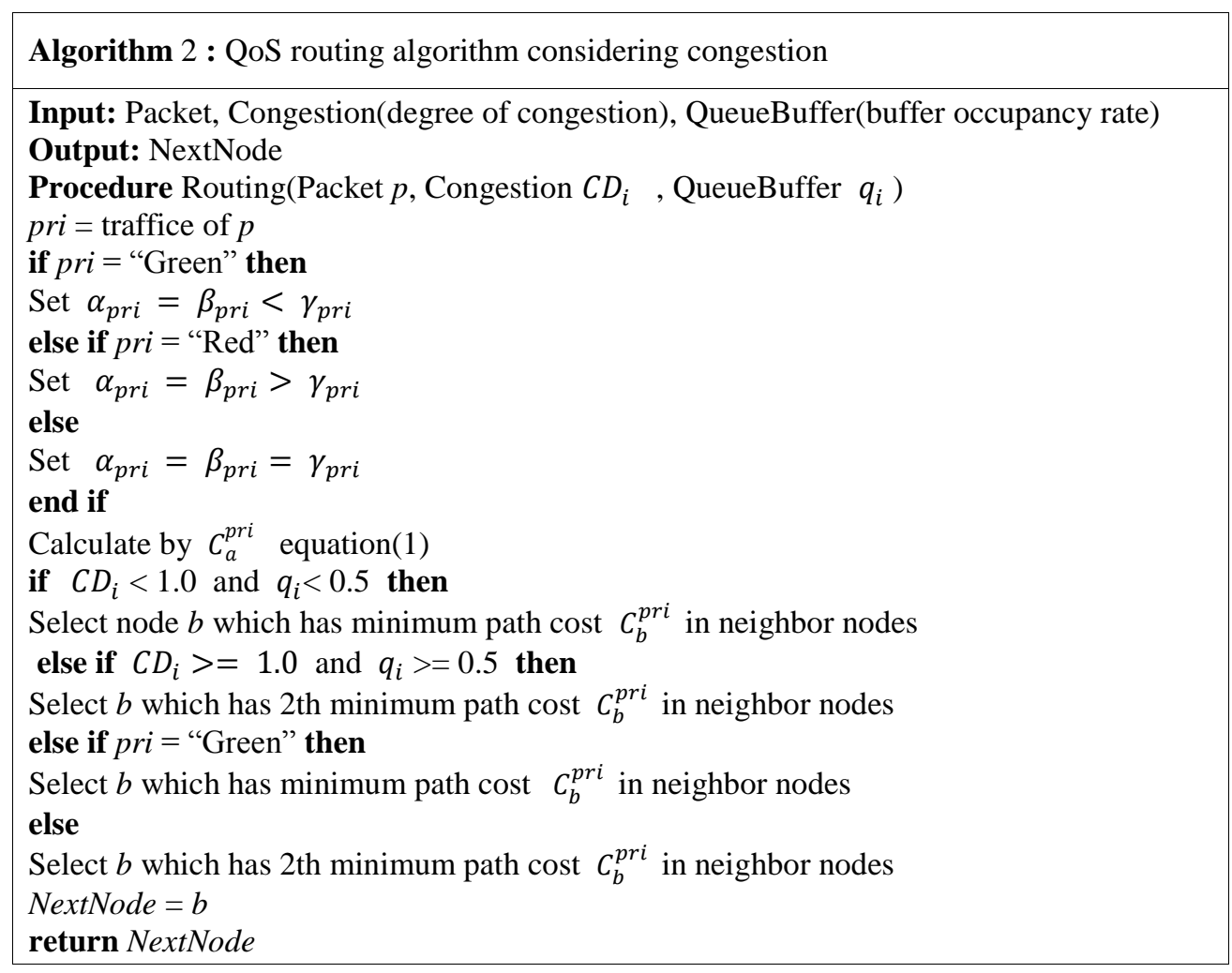

Figure 2. QoS routing algorithm considering congestion 


\section{Verification and Result Analysis}

To verify the proposed QoS routing method which considers congestion in WMSN, the performance was evaluated through simulation. The simulation was carried out using Visual Studio 2012. The simulation program was implemented with C++.

Figure 3 shows routing table information for each node as displayed on a screen in the process of simulation. Specifically, this screen presents data regarding node 97, including hop count to sink node, next forwarding node depending on packet's priorities. In addition to these, the screen also provides the information about neighbor nodes $(87,96$, and 98) including link quality, hop count to sink and path cost for each priority.

The name of first column, No in Figure 4 simply represents an order. Prionty is used for specifying packet priority. CreateTime means when the node has been created. ArrivalTime denotes the time when the packet has arrived. Delay means duration how much data is delayed. HopCount represents the number of hops existing from source node to sink node. At the bottom of the screen, useryl statistics are provided. Total Packets means the number of packets which have arrived dufing 1 second. The number of packets and delays are shown for each packet with different priority. Lastly, Average Delay is an average value of packet delay.

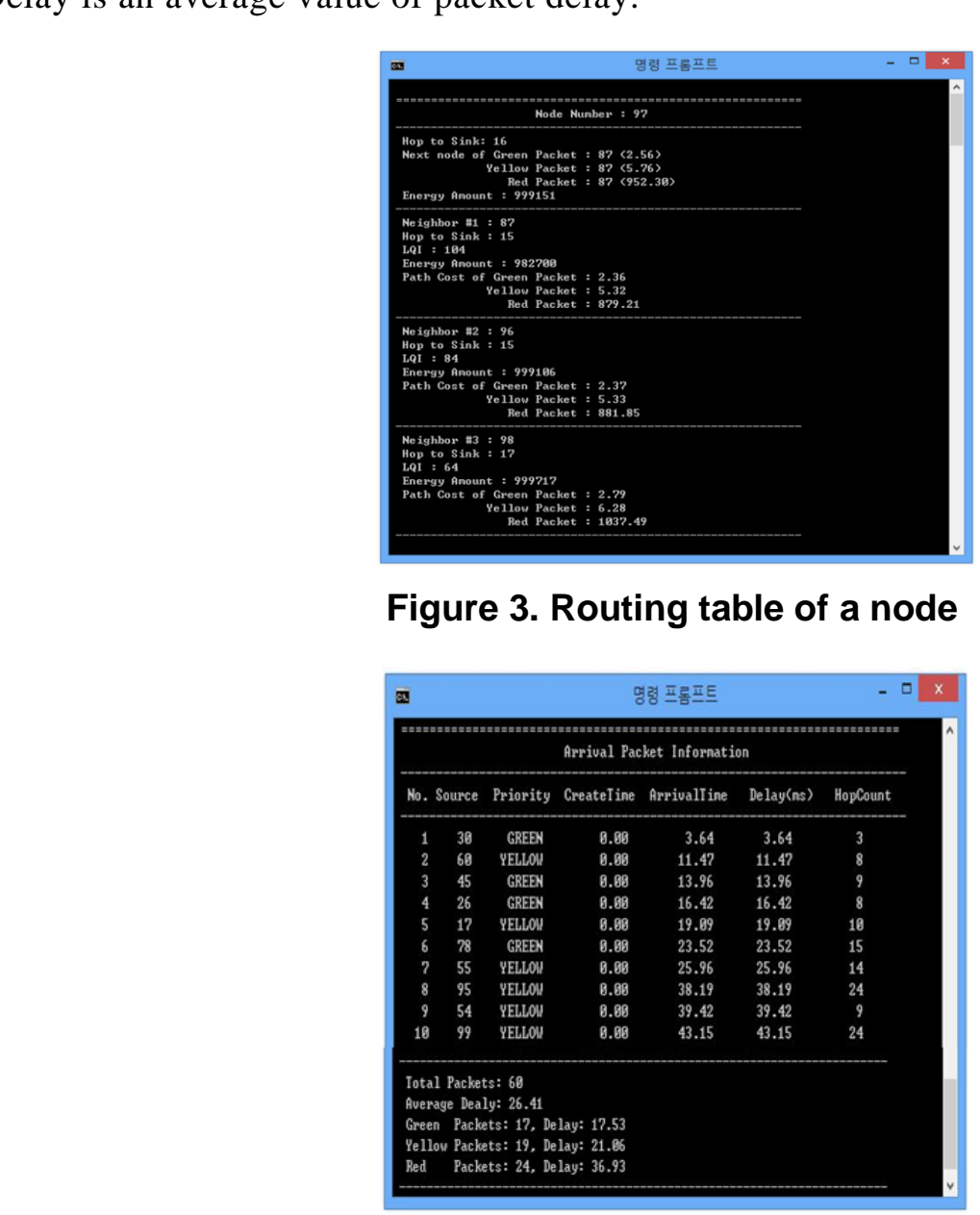

Figure 4. A screen display about packet arrival information in the process of simulation 
As a result of verification, Green packet is able to perform reliable transmission as packet loss rate and delay are reduced by being transmitted to link of high quality within the shortest distance. As red packet does not consider shortest distance and link of high quality, its packet loss or delay is considered to be considerable but this packet is effective in terms of reliability as number of packet that is lost in halfway is limited since it puts its priority on residual energy. Overall transmission rate is enhanced as Yellow packet that uses middle path between Green packet and Red packet has more room for utilizing resources. In addition, as routing algorithm for supporting QoS considering congestion situation selects main node and alternate node with minimum path cost based on packet priority, service differentiation for QoS guarantee of packet and energy efficient routing service could be supported.

\section{Conclusion}

This paper proposes an enhanced QoS routing method with considerations of congestion in WMSNs. The proposed method aims at improving packet throughput whoreas minimizing end-to-end delay as well as supporting QoS requirement In this method, data would be processed in accordance to their priority. Data with higher priority can be sent faster than data with lower priority. Thus this method enables to offer differentiated service for the network to satisfy QoS requirement. And path setting is nade by referring LQI value through which number of hop up to sink, information for residual energy OD neighbor node and quality of link could be identified based on priority mâked in source nøde.

In addition, when congestion of nodebeing selected as main path through congestion control is sensed, main path becones stand-bycondition and alternate path is operated sequentially. Where, the node being selected as alternate path is a node being selected based on minimum cost and most optimal path followed by main path node. The reason why alternate path is used is to rinlimize packet loss by congestion and reduction of delay time and data integrity. As a resuly of verification, as neighbor node is selected based on minimum path cost by each packet priority, service differentiation for ensuring QoS by packet and energy efficient routing service could be supported.

\section{Acknowledgements}

This research was supported by the MSIP (Ministry of Science, ICT and Future Planning), Korea, under the GITRC(Convergence Information Technology Research Center) support program (NIPA-2013-H0401-13-2008) supervised by the NIPA(National IT Industry Promotion Agency). This study by the "Leaders Industry-university Cooperation Project, Ministry."

\section{References}

[1] S. Ehsan and B. Hamdaoui, A survey on energy-efficient routing techniques with QoS assurances for Multimedia Wireless Sensor Networks", IEEE Comm. Surveys Tutorials, (2011), pp.1-14.

[2] I. F. Akyildiz, T. Melodia and K. R. Chowdhury, "A Survey on Wireless Multimedia Sensor Networks", The Journal of Computer Networks, vol. 51, (2007), pp. 921-960.

[3] I. F. Akyildiz, S. Weilian, Y .Sankarasubramaniam and E. Cayirci, "A Survey on Sensor Networks", Comm. IEEE, vol. 40, no. 8, (2002), pp. 102-114.

[4] C. Wang, K. Sohraby, V. Lawrence, B. Li and Y. Hu, "Priority-based Congestion Control in Wireless Sensor Networks", IEEE International Conference on Sensor Networks, Ubiquitous, and Trustworthy Computing, vol. 1, (2006). 


\section{Authors}

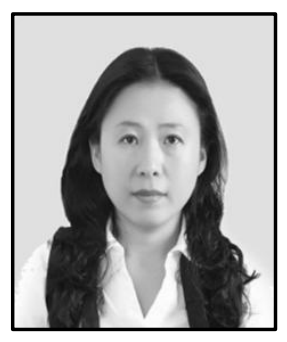

\section{Si-Yeong Bae}

She received the B.S. degree in information and communication from Seoil University, Seoul, Korea, in 2006, the M.S. degree in computer education from Hankuk University of Foreign Studies, Seoul, Korea, in 2008, and the Ph.D. student in computer science at the Sunchon National University, Suncheon, Korea, since 2009. Her current research interests include wireless sensor network, Database, routing <protocol, combinatorial optimization.

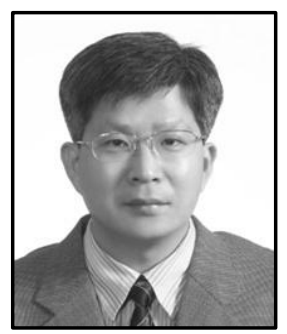

Sung-Keun Lee (Corresponding Author) •

He received the B.S., M.S., and Ph. D degrees in electronics engineering from Korea University, Seoul, Korea, in 1985, 1987, and 1995, respectively. From 1987 to 1992, he was with Samsung electronics Co., Ltd, Korea. He joined the department of Multimedia Engineering, Sunchon National University, Suncheon, Korea, in 1997, where he is currently a Professor. His research interests include energy efficient Ethernet, wirelesse Sensor network multimedia communication and Internet QoS.

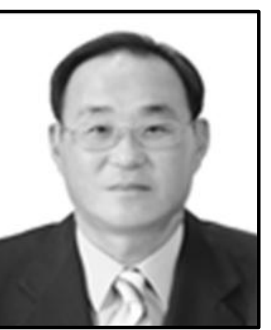

Jin-Gwang Koh

He received the B.S., M.S., and Ph. D. degrees in Computer Science and Engineering from Hongik University, Seoul, Korea, in 1982, 1984, and 1997, respectively. He was also Visiting Scientist to Oregon State University for the year 1997-1998. He was vice president of Korean Institute of Information Scientists and Engineers. He joined the department of Computer Science and Engineering, Sunchon National University, Sunchon, Korea, in 1988, where he is currently a Professor. His research interests include Database, wireless sensor networks.

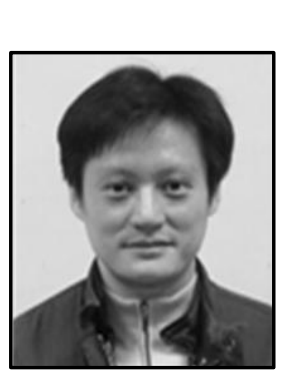

\section{Kyoung-Wook Park}

He received the B.S. degree from Sunchon National University, Suncheon, Korea, in 1997, his M.S degree from department of computer science and statistics, Chonnam National University, Gwangju, Korea, in 1999, his Ph.D. degree from department of computer science, Chonnam National University, Gwangju, Korea, in 2004. His current research interests are parallel and distribution processing, graph theory, theory of computation, bioinformatics and Augmented Reality. 
International Journal of Multimedia and Ubiquitous Engineering Vol.9, No.4 (2014)

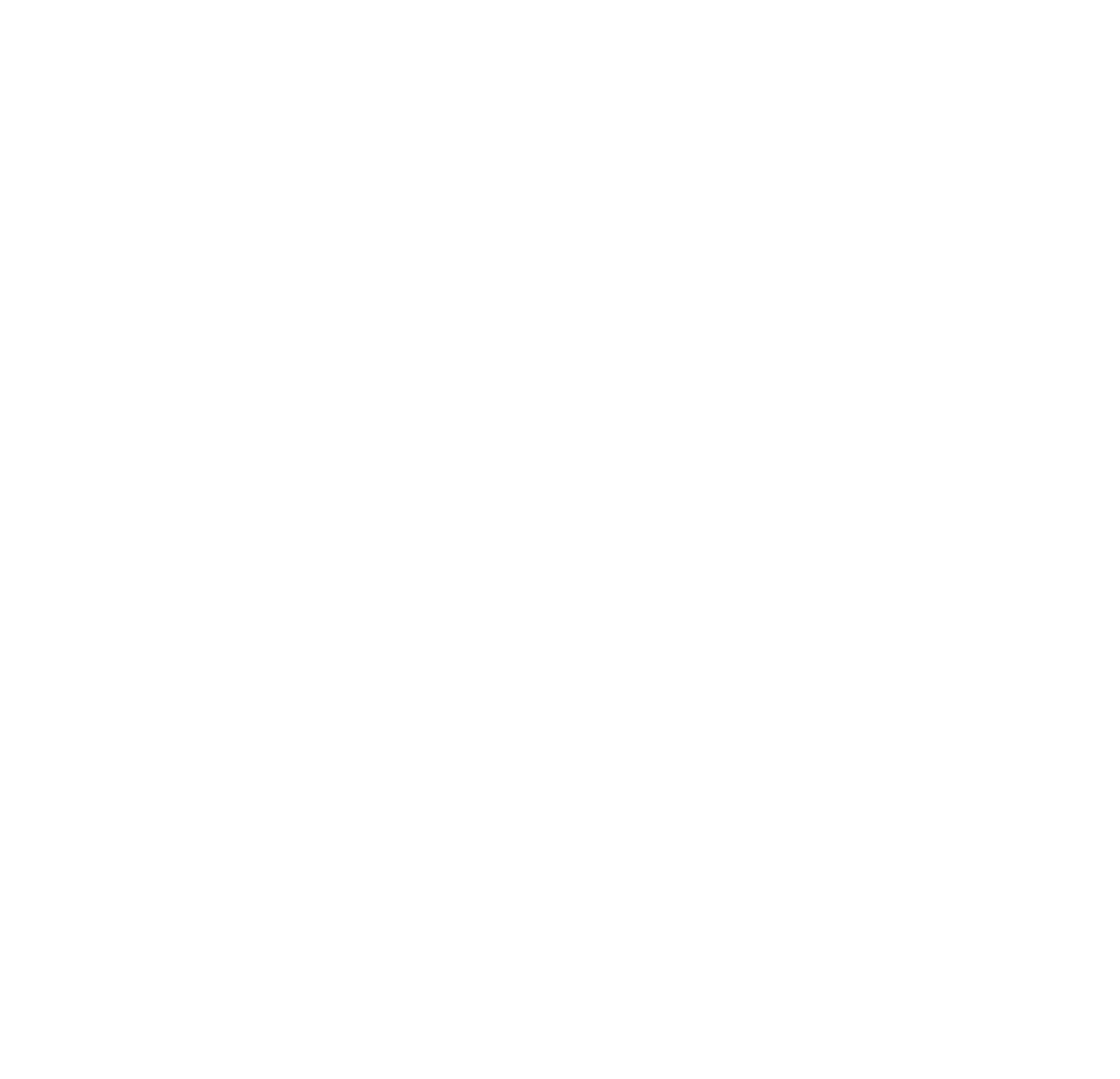

